\title{
Indicaciones de autocuidado para el adulto mayor cuidador de niños: Una mirada desde enfermería
}

Self-Care Instructions for the Elder-Caregiver of Children: A Look from Nursing

\section{Indicações de autocuidado para o idoso cuidador de crianças: Um olhar desde a enfermagem}

\author{
Liliana Quevedo-León, Enf., MSc. * \\ Herly Ruth Alvarado, Enf., MSc. **
}

\begin{abstract}
Resumen
Introducción: Según el informe de: "Envejecimiento demográfico. Colombia 1951-2020 dinámica demográfica y estructuras poblacionales", el grupo poblacional de personas mayores de 60 años es mayor que otros grupos poblacionales; este hecho plantea grandes retos para el profesional de enfermería que debe prepararse y contribuir para satisfacer las diferentes necesidades de salud de los adultos mayores, más aún si se tiene en cuenta que ellos se convierten en cuidadores de sus nietos de manera voluntaria o por compromiso, debido a los cambios en la estructura familiar dada por divorcios, migración, madres solteras, ingreso de la mujer a la vida educativa y laboral, entre otros, que han originado cambios en los roles familiares. Objetivo: Identificar cómo repercute el rol del cuidador de su nieto en la salud del adulto mayor que ejerce este papel, con el fin de
\end{abstract}

evitar o prevenir las complicaciones ocasionadas por dicha tarea. Discusión: Este rol de cuidador implica aspectos positivos y negativos en la salud del adulto mayor; negativos como dejar en segundo plano su autocuidado y su vida social; y como efecto positivo sentimientos de satisfacción como sentirse útil y productivo. Conclusiones: Aunque la condición de salud del adulto mayor que ejerce el rol de cuidador de su nieto se ve afectada tanto positiva como negativamente, en este estudio se hace énfasis en el aspecto negativo porque se puede presentar mayor riesgo de problemas emocionales, cardiovasculares y osteomusculares, así como el empleo de estrategias para disminuir tales riesgos. [Quevedo-león L, Alvarado HR. Indicaciones de autocuidado para el adulto mayor cuidador de niños: Una mirada desde enfermería. MedUNAB 2017-2018; 20(3): 362-367].

Palabras clave: Abuelos; Anciano; Niño; Enfermería; Cuidadores; Cuidado del Niño.

\footnotetext{
* Enfermera, magíster en Enfermería Salud Familiar y Atención Primaria, profesor instructor de la Facultad de Enfermería, Pontificia Universidad Javeriana, Bogotá, Cundinamarca, Colombia.

** Enfermera, magíster en Política Social, profesor asistente de la Facultad de Enfermería, Pontificia Universidad Javeriana, Bogotá, Cundinamarca, Colombia.
}

Correspondencia: Liliana Quevedo León. Carrera 7 No. 40-62, Piso 7, Pontificia Universidad Javeriana, Sede Bogotá. Teléfono: +57 (1) 3208320, extensión: 2668. E-mail: quevedo.I@javeriana.edu.co. 


\section{Abstract}

Introduction: According to the report: "Demographic aging. Colombia 1,951-2,020 population dynamics and population structures", the population group of people over 60 is older than other population groups; this fact poses great challenges for the nursing professional who must prepare and contribute to meet the different health needs of the elderly; even more so if it is taken into account that they become caregivers of their grandchildren voluntarily or by commitment due to changes in the family structure given by divorces, migration, single mothers, women's entry into educational and work life, among others, which have led to changes in family roles. Objective: To identify how the role of the caregiver of his/her grandchild affects the health of this elderly person who plays this role, in order to avoid or prevent complications caused by such task. Discussion: This caregiver role implies positive and negative aspects in the elderly's health; negative aspects such as leaving his/her self-care and social life behind, and positive ones such as feelings of satisfaction of feeling him/herself useful and productive. Conclusions: Although the health condition of the elderly person who plays the role of caregiver for his/her grandchild is affected both positively and negatively, this study lays emphasis on the negative aspect because there may be a greater risk of emotional, cardiovascular and osteomuscular problems, and also in the use of strategies to reduce such risks. [Quevedo-león L, Alvarado HR. Self-Care Instructions for the Elder-Caregiver of Children: A Look from Nursing. MedUNAB 2017-2018; 20(3): 362-367].

Keywords: Grandparents; Aged; Child; Nursing; Caregivers; Child Care.

\section{Introducción}

Las estadísticas evidencian que el 70\% de las mujeres mayores de 65 años han cuidado a sus nietos o los cuidan en la actualidad. De las abuelas que los cuidan actualmente, casi la mitad lo hace a diario, como si se tratara de un trabajo fijo (1). Para el año 2000 en los Estados Unidos, más de 2.4 millones de adultos mayores se encontraban cuidando a sus nietos (1). En Colombia los datos reportados por el Departamento Administrativo Nacional de Estadística de Colombia (DANE) deja ver que un 13\% de parejas delegan el cuidado de sus hijos a familiares cercanos, en especial a sus padres, quienes con su compromiso con el cuidado del nieto brindan mayor seguridad a los padres permitiendo de esta manera la migración (2).

Tal es la realidad vivida por abuelos que asumen el rol de cuidador, ya sea para ayudar a sus hijos en la crianza o porque al estar dependiendo económicamente de ellos, tienen que hacerlo. Se trata de abuelos que por lo general forman parte del grupo de adultos mayores, es decir, mayores de sesenta años.

La tarea de cuidar puede llevar a los adultos mayores a experimentar, como se mencionó anteriormente, efectos negativos que implican tener menos tiempo para ellos

\section{Resumo}

Introdução: De acordo com o relatório: "envelhecimento demográfico. Colômbia 1951-2020 dinâmicas demográficas e estruturas populacionais", a população com mais de 60 anos é maior do que outros. Esse fato, coloca grandes desafios para o profissional de enfermagem que deve preparar e contribuir para atender as diferentes necessidades de saúde dos idosos, ainda mais se se considera que eles se tornam cuidadores de seus netos voluntariamente ou por compromisso, devido à mudanças na estrutura familiar dada por divórcios, migração, mães solteiras, entrada de mulheres na vida estudantil e profissional, entre outras, que mudam os papéis na estrutura das familias. Objetivo: Identificar, como o cuidado de seu neto, afeta a saúde do adulto mais velho que exerce esse papel, a fim de evitar ou prevenir as complicações causadas por esta tarefa. Discussão: Este papel do cuidador implica aspectos positivos e negativos na saúde dos idosos; negativa como deixando em segundo plano seu autocuidado e sua vida social; e como um efeito positivo, sentimentos de satisfação, como sentir-se útil e produtivo. Conclusões: Embora o estado de saúde do adulto mais velho que desempenha o papel de cuidador para seu neto seja afetado de forma positiva e negativa, neste estudo o aspecto negativo é enfatizado porque pode haver maior risco de problemas emocionais, cardiovasculares e músculo-esqueléticos, bem como o uso de estratégias para reduzir esses riscos. [Quevedo-león L, Alvarado HR. Indicações de autocuidado para o idoso cuidador de crianças: Um olhar desde a enfermagem. MedUNAB 2017-2018; 20(3): 362-367].

Palavras-chave: Avós; Idoso; Criança; Enfermagem; Cuidadores; Cuidado da Criança.

mismos y su autocuidado, pérdida de libertad para realizar actividades con sus pares, estrés y sentimientos de angustia frente a la responsabilidad del cuidado de sus nietos. Estudios evidencian que si el cuidado implica varias horas al día o se realiza en forma permanente, se aumenta el riesgo de ataques cardiacos, depresión, tristeza debido no solo al tiempo sino a las circunstancias familiares que atraviesan, falta de recursos económicos y algunos conflictos se pueden generar frente al cuidado del adolescente (3).

Estudios muestran que adultos mayores cuidadores perciben deterioro en su salud en comparación con los adultos mayores no cuidadores, debido a la sobrecarga que conlleva menos descanso, menor actividad recreativa, menor interacción social; lo cual afecta su salud mental y física (4). Sin embargo, el ejercer el rol de cuidador permite a este grupo poblacional tener sentimientos positivos ya que les permite sentirse útiles, seguros, desarrollar relaciones afectivas sólidas con sus nietos, con sus hijos y, en ocasiones, los lleva a mejorar estilos de vida por su nueva responsabilidad frente al cuidado y el ejemplo que son para sus nietos.

Reconociendo que el cuidar tiene implicaciones en la salud física y emocional, sumado a factores sociales y en ocasiones económicos, se hace importante reforzar que el 
cuidado de los nietos no es responsabilidad de los abuelos y si no se siente capaz o en la disponibilidad de hacerlo tiene toda la libertad para negarse. Si a pesar de no querer asumir el cuidado y lo hace por la necesidad de contribuir con sus hijos, el profesional de enfermería debe realizar un abordaje íntegro y brindarle herramientas para disminuir los efectos negativos que pueda acarrear esta responsabilidad.

En el caso de adultos mayores cuidadores de sus nietos, el profesional de enfermería debe asumir su liderazgo con énfasis en el cuidado del adulto mayor para proponer programas de prevención y promoción de la salud indicando la importancia del autocuidado, el descanso y autonomía en relación a la decisión de cuidar y la importancia de compartir tiempo con pares y grupos de apoyo (2). Este artículo plantea una reflexión a través de la búsqueda de literatura derivado de un interés investigativo de la repercusión en la salud del adulto mayor en su rol de cuidador, en su mayoría ejercido por las abuelas.

\section{Acerca del cuidado}

El cuidado existe desde el inicio de la vida; el ser humano como ser vivo necesita ser cuidado, porque se trata de una acción que permite la continuidad de la vida. Así como hay personas que necesitan atención, existen quienes proporcionan cuidados a otros en las distintas etapas de la vida; en consecuencia, el cuidar y ser cuidado es imprescindible para la vida y la perpetuidad del ser humano (3). Entre los ciclos de la vida, la niñez es una de las etapas que más requiere cuidado por las características que implica este ciclo vital.

Para hablar del cuidado del niño se debe mencionar primero quién es el niño; la Convención sobre los Derechos del Niño (CDN) aprobada desde el 20 de noviembre de 1989 definió niño de la siguiente manera: "se entiende por niño todo ser humano menor de dieciocho años de edad, salvo que, en virtud de la Ley que le sea aplicable, haya alcanzado antes la mayoría de edad"; el cuidado de las niñas, los niños y los adolescentes, ha adquirido un principal interés desde que en la legislación se les reconoce como sujetos de derechos. El Código de la Infancia y la Adolescencia colombiano, expedido el 8 de noviembre de 2006 (4), demanda la formación de personas capaces y promueve como el cuidado es responsabilidad de la familia, la sociedad y el Estado.

Por sus características el niño es dependiente y requiere de cuidado permanente en sus diferentes etapas; en general los primeros y principales cuidadores son los padres quienes deben brindar a sus hijos la garantía de acceso y cumplimiento de derechos, y la protección ante las amenazas y riesgos que les impidan su buen desarrollo en la sociedad.

El concepto de cuidado discutido en las ciencias sociales significa: acción y pensamiento, atención y esmero, atención sostenida o intensiva, acción con conciencia y reflexión, actitud de preocupación, actividad cognitiva y emocional entre el cuidador o la cuidadora y la persona que, por su condición de vulnerabilidad, requiere ser cuidada(5). Generalmente, la familia ha sido y seguirá siendo la primera institución que proporciona cuidado en situaciones de dependencia y la mujer siempre ha estado ligada a la permanencia en casa, al cuidado de los hijos y de las tareas domésticas, tal vez por estos motivos, por cultura y tradición, habitualmente es ella quien proporciona cuidado de manera continua, asignándosele el rol de cuidadora $(3,6)$.

En Colombia para el año 2008 había un 29.8\% de hogares con autoridad femenina y para el año 2012 aumentó al $34.4 \%$ (7), estos cambios en la estructura familiar y el ingreso de la mujer al estudio y a la vida laboral, han dado paso a que los abuelos se conviertan en cuidadores de sus nietos (8), la dedicación ha dejado de ser esporádica y voluntaria, para convertirse en obligatoria, incluso muchos de ellos están aceptando la responsabilidad de criar a sus nietos cuando los propios padres no quieren hacerlo.

Como se ha mencionado, el cuidado brindado a los nietos por lo general recae en las mujeres de la familia, el cuidado que realizan las abuelas es frecuente e incluso se ha denominado "Cadenas Globales del Cuidado" (9). Cuidar para las abuelas significa ayuda, solidaridad y es considerado como un deber implícito; no obstante, hay factores propios de ellas que pueden afectar el que asuman o no este nuevo rol de cuidadora entre los que se puede mencionar el nivel educativo; se ha evidenciado que entre más alto nivel de estudio tenga es menor la probabilidad que cuide el nieto ya que lo más seguro es que sea activa laboralmente y cuente con menos tiempo para cuidar (8). Por otro lado, el que los padres de los niños tengan un bajo ingreso económico también aumenta las probabilidades de restringir el cuidado de los niños a la abuela ya que es menor el compromiso de remuneración con ella convirtiéndose en una cuidadora informal.

Varios estudios coinciden en afirmar que cuando la abuela cumple el rol de cuidadora de sus nietos por falta de padres, se incrementa el riesgo de que presente problemas físicos y mentales (10) aumentando el riesgo de enfermedad coronaria en la mujer(11) y en ocasiones también representa un esfuerzo económico por parte de los adultos mayores (12), no siendo lo mismo cuidar a un bebe que a un adolescente, un nieto que dos o más, unas pocas horas a la semana o varias horas al día; todos estos factores influyen a la hora de asumir el cuidado.

Algunos autores han evidenciado que si la carga de cuidado en el adulto mayor es grande se reflejan sentimientos de tristeza, aburrimiento y soledad (2). Se podría considerar que estos sentimientos ocurren porque el adulto mayor pierde su núcleo de amigos, al igual que se restringe el tiempo y libertad para estar fuera del hogar, inclusive se restringe la asistencia a sus controles y/o seguimientos médicos por falta de tiempo. 
La literatura muestra que el ser cuidador también es un factor de riesgo para morir y se ha encontrado un $63 \%$ más de mortalidad en las personas cuidadoras; en este sentido, se encontró que las tasas de mortalidad fueron más altas entre las personas con alguna enfermedad prevalente $(22.5 \%)$, seguidos de aquellas que presentaban enfermedad subclínica (11.6\%), y finalizaba con los que no presentaban ninguna enfermedad (5.4\%) (13). No se debe olvidar que por lo general se llega a la vejez con una o varias Enfermedades Crónicas No Transmisibles (ECNT), las cuales necesitan de control y seguimiento periódico, así como adoptar estilos de vida saludables que implican tiempo para recreación, tiempo para actividad física, tiempo para desplazamiento a exámenes y/o reclamar medicamentos, actividades que muchas veces se distancian o se suspenden por estar al cuidado de los nietos. Estudios concluyen que los abuelos obligados a asumir la responsabilidad de encargarse del cuidado de sus nietos debido a problemas de sus hijos como: drogadicción, violencia, encarcelación, etc. (14) pueden tener peor pronóstico de salud a diferencia de los que eligen cuidar a sus nietos por decisión propia algunas horas a la semana, sin obligación, o que pueden negarse al cuidado si surge algún imprevisto o si no lo desean.

Contrario a lo expuesto, se evidencia en la literatura que el cuidar nietos puede mejorar la salud (15), algunos autores refieren que el cuidar proporciona sentimientos de utilidad y refiere que si los adultos mayores no tuvieran a cargo ese cuidado muy poco seria el contacto con sus nietos (16). A nivel familiar, el cuidado dado por los abuelos produce ventajas para los padres del niño, entre estos menores costos y para ellos como cuidadores, sentirse útiles tanto por la relación con los nietos, como el hecho de sentirse sujetos de valor indispensable en la familia (14); de igual manera, según Calderón, algunos adultos mayores que cuidan a sus nietos refieren que rejuvenecen, que disfrutan (17) esta relación y disminuyen sentimientos como la soledad y el aburrimiento(18).

\section{Implicaciones para el adulto mayor}

El cuidar y el ser cuidado tiene implicaciones, los abuelos que cuidan revelaron sentimientos antagónicos, ya que se pueden mezclar la satisfacción y orgullo frente al cuidado por sentirse útiles y productivos al ayudar a sus hijos en la crianza de sus nietos y en ocasiones labilidad emocional por el estrés, y la irritabilidad por la gran responsabilidad(2).

Se encontró bibliografía que plantea como este evento genera sucesos positivos y negativos para el adulto mayor (19). Para un gran número de abuelos el compromiso de cuidar a sus nietos se convierte en una responsabilidad durante varias horas del día lo que limita el tiempo para sí mismo, su autocuidado y la relación con sus pares; estas situaciones pueden generar tensión, estrés y aislamiento social, en algunas ocasiones incluye la responsabilidad económica lo que puede desarrollar depresión en el adulto mayor $(20,21)$. La edad del nieto puede también estar condicionando la sensación de estrés debido al manejo que deben dar a distintas situaciones: desde la irritación o pataleta del niño de tres años, hasta los problemas de drogas, rebeldía o incidentes que puede presentar un adolescente (22). Estas situaciones llevan a que el adulto mayor asuma y enfrente compromisos en búsqueda del bienestar de su nieto, también temor a sufrir un fracaso frente a la crianza que le ha sido delegada. El cuidar implica en estos casos para el adulto mayor estrés y depresión que repercutirán directamente sobre su salud, incluso ocasionando el aplazamiento o cancelación de citas médicas programadas para el cuidado de su condición de adulto mayor por la responsabilidad del cuidado.

En cuanto a la parte gratificante de brindar cuidado, los abuelos pueden sentir satisfacción ya que se sienten útiles y queridos cuando perciben que el cuidar a su nieto le puede ayudar a mejorar sus conflictos y les brinda seguridad. Erikson y Kivnick denominaron este aspecto como una "segunda oportunidad en la generatividad" y fue planteado también en la teoría de la continuidad (15). Otro factor positivo para los abuelos cuidadores es que puede modificar en ellos algunas conductas inapropiadas y puede intervenir en adquirir comportamientos saludables como dejar de fumar o beber por la responsabilidad que sienten de dar un buen ejemplo y de mostrar estilos de vida saludables (23), además pueden hacer sentir a estos adultos mayores que su red de apoyo aumenta al contar con su nieto. Esta sensación de satisfacción está más presente en los abuelos que realizan la tarea de cuidar de forma altruista y no por obligación.

\section{Aspectos negativos para el adulto mayor}

El cuidar no solo implica entonces responsabilidad sino también actividad física. Dependiendo del tiempo que se dedique al niño puede generar mayor o menor grado de cansancio físico y mental (24). Algunos abuelos manifestaron en un estudio el agotamiento y desgaste que ocasionaba el comportamiento de sus nietos. Expresaron la presencia de mialgias cuando cuidaban a un nieto menor y cansancio mental, agotamiento y desgaste sobre todo cuando los cuidados iban dirigidos a adolescentes (9), se desplegaron también sentimientos de tensión, estrés y depresión algunos provocados por los problemas que tenían sus hijos como detención de alguno de los padres, problemas de drogadicción, alcoholismo, separación o enfermedades como el Virus de Inmunodeficiencia Humana (VIH) (19).

En ocasiones, la responsabilidad económica del nieto recae sobre los abuelos, lo que aumenta el riesgo para que sientan depresión, ya que algunas veces no cuentan con ingresos económicos fijos o suficientes. Otra modificación sufrida por estos cuidadores es en el patrón de sueño y descanso, ya que declararon frecuentemente la presencia de insomnio por 
la misma sensación de sobrecarga de trabajo y la sensación de celos constantes por parte de sus hijos frente al manejo de la autoridad con el niño y el desplazamiento afectivo, lo que en ocasiones genera conflicto.

Por otra parte, al responder por el nieto y tener que asistir al colegio evidencia diferencia generacional con los padres de los otros niños y esto provoca de alguna manera aislamiento social. El estudio: presentado por Sunmin Lee, ScD, Graham Colditz, MD, DrPH, Lisa Berkman, planteó que cuidar más de 9 horas semanales al niño puede aumentar el riesgo de sufrir un ataque cardiaco (11).

\section{Beneficios de las relaciones intergeneracionales}

Que el cuidado lo realicen los abuelos, genera una solidaridad intergeneracional que puede procurar oportunidades de conocimiento, ayuda y apoyo entre abuelos y nietos, este aspecto ha tomado una relevancia especial por las circunstancias actuales del núcleo familiar (18).

El papel socializador que desempeñan los abuelos a veces pasa desapercibido, desconociendo la importancia que tiene la relación que surge en este binomio. El crecer y vivir en un ámbito donde se siente ser querido, provoca en el individuo un cambio positivo que puede aportar mucho a la sociedad.

Marks plantea que los abuelos pueden ser un punto de apoyo importante para el niño en la sociedad actual, porque brindan estabilidad emocional, financiera y reforzamiento de valores cuando hay problemas familiares como: soledad por la necesidad de salir los dos padres a trabajar, divorcios, drogas, abuso del alcohol y enfermedades. En ocasiones los abuelos pueden funcionar como negociadores entre nieto y padre disminuyendo las tensiones que se pueden generar entre ellos (15). Otro aspecto importante en esta relación es la transmisión de la cultura a partir de prácticas realizadas por el adulto mayor, juegos, música y costumbres que el niño conoce mediante el cuidado (doble dependencia). Otro aspecto a destacar es que puede ocurrir un desplazamiento afectivo donde el niño reconozca mayor afecto hacia el adulto mayor que lo cuida, que por sus padres(16).

\section{Cuidados de Enfermería}

Para disminuir los efectos negativos en el adulto mayor cuidador es importante estimular el no abandonar su autocuidado, apoyándolo para que asuma la responsabilidad individual en el manejo de su estado de salud, diseñando en conjunto con el personal de enfermería un plan de cuidado, que incluya metas educativas y conductas adecuadas en pro de alcanzar un óptimo nivel de bienestar e inclusive recalcando la importancia de aprender a decir no cuando sea preciso. Generalmente, el adulto mayor presenta alguna enfermedad crónica no transmisible que demanda una buena adherencia o simplemente requiere de un control periódico de su salud, que puede ir postergando por estar a cargo del cuidado de sus nietos. Se debe sensibilizar al adulto mayor sobre la necesidad de tener un cuidado adecuado con su salud ya que de ello depende la posibilidad de ser cuidador.

El cuidar requiere desarrollar mayor actividad física por esta razón se deben enseñar técnicas de buena postura, alzar de forma adecuada a los nietos sin que se vea en riesgo de presentar espasmos musculares o problemas de columna. Se debe estimular para que realice actividades que sean agradables tanto para él como para el nieto, por ejemplo: realizar algún deporte, caminar, nadar o compartir juegos de mesa. Entre otras actividades se puede programar visitas a museos, parques, exposiciones de arte, obras de teatro o cines. Es necesario estimular también un adecuado descanso y autocuidado, como tener horas suficientes de sueño y de espacios para compartir con sus pares.

El cuidar sobre todo a nietos adolescentes o de personalidad difícil, implica mayor riego para presentar estrés; en caso de sentirse agobiado o cansado, debe buscar con quien poder hablar para lograr desahogarse y así eliminar toda esta tensión; en ese caso puede contar con apoyo de un amigo o familiar, la iglesia o un profesional de salud, es decir que desde enfermería se deben desarrollar estrategias para mejorar las redes sociales.

\section{Conclusiones}

El cuidado de los hijos a través de la historia y culturalmente ha sido asumido por la familia y en especial la mujer; sin embargo, el contexto mundial en el que se desenvuelve como por ejemplo: mayor educación, desempeño laboral fuera, sustento económico de la familia como cabeza de hogar, los divorcios, la necesidad de migrar a otros países para mejorar las condiciones de vida, han repercutido en que el cuidado de los niños se desplace a los abuelos, quienes dejan de ser sujetos necesitados de atención y cuidado para convertirse en sujetos cuidadores.

El adulto mayor que desempeña el rol de cuidador de sus nietos puede presentar sentimientos ambiguos como alegría, amor, depresión y estrés frente a la responsabilidad; este rol genera también efectos positivos sobre todo en los que cuidan a sus nietos por gusto y no por compromiso considerando esta tarea como gratificante que les produce mayor vitalidad y les permite tener un envejecimiento activo.

Así mismo, el cuidado que realiza el adulto mayor a cargo de su nieto ofrece ventajas y desventajas para el cuidador, frente a las desventajas es importante enseñarles estrategias para disminuir riesgos tanto físicos como emocionales, como enseñar técnicas de buena postura, el reforzar que realice 
algún deporte, el que priorice tiempo para el descanso y el compartir con amigos. Además, las características y la edad del niño son importantes para tener en cuenta ya que tienen implicaciones en la salud; el cuidar a un niño pequeño puede generar más riesgos físicos ocasionados por la actividad física que debe realizar durante el cuidado, mientras que el cuidado de adolescentes puede ocasionar mayores problemas de estrés.

Los adultos mayores cuidadores presentan mayor riesgo de tener problemas emocionales, cardiovasculares y osteomusculares entre otros; para evitar que su salud se vea afectada el profesional de enfermería debe buscar estrategias buscando disminuir riesgos como por ejemplo: educar sobre la importancia de tener tiempo libre con sus pares, necesidad de descanso y autocuidado, mantener posturas y movimientos adecuados para evitar riesgos osteomusculares. Es necesario estimular las redes de apoyo sin dejar de lado que se debe enseñar al adulto mayor a no sacrificar su bienestar y fomentar en ellos el no abandonar su autocuidado. Por último, es claro que el niño que es cuidado por sus abuelos generalmente se ve beneficiado y desarrolla confianza y seguridad.

\section{Conflicto de intereses}

Los autores declaran no tener conflicto de interés.

\section{Referencias}

1. Diario el Pais. Casi la mitad de las abuelas que cuidan a sus nietos lo hace a diario. Edición impresa. EL PAís. 2013(7/10/2013).

2. MartínezA. Aproximación a los conflictos generados entre los abuelos cuidadores de nietos y los padres en la sociedad actual. [internet]. 2010. [Revisado el: 7/09/2013]. Disponible en: http://www.eumed.net/rev/ cccss/10/almm.htm.

3. Vaquiro S, Stiepovich J. Cuidado informal, un reto asumido por la mujer. Ciencia y enfermería. 2010; 16(2):9-16.

4. Procuraduría General de la Nación. Código de la infancia y la adolescencia. [internet]. 2006. [Revisado el: 7/09/2013]. Disponible en: http://apps.mintrabajo.gov.co/ siriti/info/codigo_infancia_y_adolescencia_ley_1098_de 2006_comentado.pdf.

5. Puyana Y. Migración y familias. [internet]. 2013. [Revisado el: 7/09/2013]. Disponible en: http://www.humanas.unal. edu.co/migracionyfamilias/contactos/yolanda-puyana/.

6. Larrañaga I, Martín U, Bacigalupe A, Begiristáin J, Valderrama M, Arregi B. Impacto del cuidado informal en la salud y la calidad de vida de las personas cuidadoras: análisis de las desigualdades de género. Gaceta Sanitaria. 2008; 22(5):443-450.

7. Medellín Como Vamos. Encuesta de Calidad de Vida del DANE revela algunos datos interesantes de los hogares colombianos en 2012. [Internet]. 2013. [Revisado el: 07/09/2013]. Disponible en: https://www. medellincomovamos.org/.

8. Partidas R. Trabajadoras de la electrónica en Jalisco: las abuelas como proveedoras de cuidado infantil. El cotidiana. 2004; 19(125):68-77.

9. Micolta A, Escobar A. Si las abuelas se disponen a cuidar, madres y padres pueden emigrar. [Internet]. 2013. [Revisado el: 30/06/2013]. Disponible en: http://www.scielo.org.ve/pdf/rvem/v15n35/art06.pdf.

10. Weisbrot M. ¿Las abuelas que cuidan nietos tienen más riesgo de enfermarse? Evid actual. 2007; 10(6): 188-191.

11. Lee S, Colditz G, Berkman L, Kawachi I. Caregiving to children and grandchildren and risk of coronary heart disease in women. Am J Public Health. 2003; 93(11):1939-1944.

12. Mestre J, Guillén J, Caro F. Abuelas cuidadoras en el siglo XXI: recurso de conciliación de la vida social y familiar. Portularia. 2012; 12:231-238.

13. Schulz R, Beach $S$. Caregiving as a risk factor for mortality: the Caregiver Health Effects Study. JAMA. 1999; 282(23): 2215-2219.

14. Triadó C, Villar F, Solé C, Celdrán M, Pinazo S, Conde L, et al. Las abuelas/os cuidadores de sus nietos/as: tareas de cuidado, beneficios y dificultades del rol. INFAD Revista de Psicología. 2008; 4(1):455-464.

15. Sands R, Goldberg R, Thornton P. Factors associated with the positive well-being of grandparents caring for their grandchildren. J Gerontol Soc Work. 2005; 45(4):65-82.

16. Plá N, López M. Doble dependencia: abuelos que cuidan nietos en España. EKAINA. [Internet]. 2011. [Revisado el: 06/09/2013]. Disponible en: www.zerbitzuan.net/ documentos/zerbitzuan/Doble\%20dependencia.pdf.

17. García C, Vega C. Relaciones abuelos-nietos: una aproximación al rol del Abuelo. Sociedad y Utopía.Revista de Ciencias Sociales 2013; (41):464-482.

18. Bernal J, de la Fuente R. Relevancia psico-socio-educativa de las relaciones generacionales abuelo-nieto. Revista española de pedagogía. 2008; 66(258):103-118.

19. Weisbrot M, Giraudo N. Conceptos y percepciones de las abuelas sobre el cuidado de sus nietos. Estudio cualitativo en una población del Hospital Italiano de Buenos Aires. Arch Argent Pediatr. 2012; 110(2):126-131.

20. Blustein J, Chan S, Guanais F. Elevated depressive symptoms among caregiving grandparents. Health Serv Res. 2004; 39(6):1671-1689.

21. Musil C, Warner C, Zauszniewski J, Wykle M, Standing T. Grandmother Caregiving, Family Stress and Strain, and Depressive Symptoms. West J Nurs Res. 2008; 31(3):389-408.

22. Davidhizar R, Bechtel G, Woodring $B$. The changing role of grandparenthood. J Gerontol Nurs. 200; 26(1):24-9.

23. DANE. Encuesta de Calidad de Vida 2010. [internet]. 2013. [Revisado el: 7/9/2013]. Disponible en: http://www.dane.gov.co/index.php?option=com_content $\&$ view $=$ article\&id $=1159$ \& $\mid$ temid $=66$.

24. Fuller E. Canadian First Nations grandparents raising grandchildren: a portrait in resilience. Int J Aging Hum Dev. 2005; 60(4):331-42. 